\title{
CONDICIÓN DE LOS ARRECIFES DE CORAL FRENTE A COSTAS CON ASENTAMIENTOS HUMANOS Y APORTES TERRÍGENOS: EL CASO DEL LITORAL HABANERO, CUBA
}

\author{
Hansel Caballero ${ }^{*}$, Pedro M. Alcolado ${ }^{2}$ y Aloyma Semidey ${ }^{2}$
}

\begin{abstract}
RESUMEN
Se evaluó la condición de los arrecifes de coral del norte de las provincias habaneras (NW Cuba), área con influencia variable de asentamientos humanos y de aporte terrígeno, con la aplicación de indicadores biológicos del bentos de la metodología AGRRA: cubrimiento vivo de coral; densidad, diámetro máximo, mortalidad reciente y antigua y reclutamiento de corales duros; densidad de erizos Diadema antillarum y cubrimiento por macroalgas. Se muestrearon las comunidades de corales de 11 sitios de crestas arrecifales y 22 de arrecifes frontales durante el año 2004. Se calcularon los índices de heterogeneidad de Shannon y de equitatividad de Pielou. Se identificaron 33 especies de corales (mayores de 10). Hubo diferencias significativas entre sitios en los indicadores biológicos analizados que sugieren una marcada heterogeneidad en las condiciones medioambientales a lo largo de la zona de estudio. El cubrimiento medio de coral vivo en las crestas fue de $28 \%$ y en los arrecifes frontales, de $21 \%$. La densidad media en las crestas fue de 9 colonias $/ 10 \mathrm{~m}$, mientras que en los arrecifes más profundos, 12 colonias/10 $\mathrm{m}$. Se observaron poca mortalidad reciente y bajos porcentajes de corales enfermos. La mortalidad antigua se debe aparentemente a la acumulación histórica de afectaciones, tanto naturales como antrópicas. Los arrecifes estuvieron perturbados además por cubrimientos significativos de macroalgas carnosas aparentemente causados por una combinación de exceso de nutrientes e insuficiente herbivorismo. Se encontraron arrecifes en buenas condiciones posiblemente dado por cierto nivel de heterotrofía favorecido por enriquecimiento de materia orgánica particulada proveniente de la tierra.
\end{abstract}

Palabras claves: Diversidad, abundancia, arrecifes coralinos, Cuba.

\begin{abstract}
Coral reef condition north of the Havanan provinces (NW Cuba), an area with variable influence from human settlements and runoff, was assessed using the AGRRA methodology's benthic biological indicators: live coral cover, coral density, maximum colony diameter, old and recent coral mortality, coral recruitment, Diadema antillarum density, and macroalgae cover. Coral communities at 11 reef crest and 22 forereef sites were sampled during 2004. The Shannon heterogenety and Pielou evenness indices were calculated. A total of 33 species of coral, larger than $10 \mathrm{~cm}$ in maximum diameter, were identified. The biological indicators analyzed showed significant differences among sites, which suggest a marked heterogeneity in environmental conditions along the study area. The average live coral cover was $28 \%$ on crests, and $21 \%$ on
\end{abstract}

1 Acuario Nacional de Cuba, Avenida 1ra. y 60, Playa, CP 11300, Ciudad Habana, Cuba.

2 Instituto de Oceanología, Ave. 1ra., No. 18406, Rpto. Flores, Playa, La Habana, Cuba.

* hanselc@acuarionacional.cu

Recibido 8-I-2009

Aceptado 22-IX-2009

DOI: http://dx.doi.org/10.15359/revmar.1.3 
forereefs. The average densities were 9 colonies $/ 10 \mathrm{~m}$ in crests, and 12 colonies $/ 10 \mathrm{~m}$ in the deeper sites. Little recent mortality and low diseased coral percentages were observed. Old mortality is apparently due to the historical accumulation of both natural and anthropogenic effects. Reefs were also perturbed by considerable fleshy macroalgal cover, apparently a consequence of both excess of nutrients and insufficient herbivory. Reefs in good condition were found possibly due to some level of heterotophy from organic matter enrichment derived from land sources.

Keywords: Diversity, abundance, coral reefs, Cuba.

\section{INTRODUCCIÓN}

Al comienzo del siglo, alrededor del $27 \%$ de los arrecifes del mundo presentaban un alto riesgo de mortalidad; $30 \%$, un riesgo medio; y solamente $40 \%$ eran estables o tenían un bajo riesgo de destrucción (Borneman, 2001). Muchos arrecifes del Caribe han perdido hasta el $80 \%$ de la cobertura viva de coral durante los últimos 50 años (Gardner et al. 2003). El cambio climático mundial (causante de eventos masivos de blanqueamiento y huracanes de gran intensidad) y los impactos humanos (sobrepesca, contaminación, incremento de las tasas de sedimento y nutrientes provenientes de la agricultura y vertimientos urbanos) han sido las causas principales de esta degradación mundial (Wilkinson y Souter, 2008).

Los arrecifes de Cuba han sufrido igualmente deterioro tanto por causas antrópicas como naturales (Alcolado et al. 1997, 2000a, 2000b; Lang et al. 1998; Woodley et al. 2000; Jones et al. 2004, 2008). Poblaciones de coral dañadas por huracanes, brotes letales de enfermedades y eventos de blanqueamiento de grado significativo e incremento de organismos bioerosionantes por exceso de nutrientes, han creado desbalances en el proceso de erosión-calcificación, y se observan zonas arrecifales con crestas degradadas convertidas en bajos rocosos con corales aislados y arrecifes frontales con mortalidad parcial y alta cobertura algal (González-Ferrer et al. 2004).

La estrecha plataforma de la costa norte de la provincia de La Habana (que incluye la Ciudad de La Habana), ubicada en la región occidental de Cuba, presenta arrecifes de coral con variable desarrollo estructural. Estos arrecifes reciben regularmente tormentas invernales, con menor frecuencia impactos de huracanes; y con diferente grado de magnitud, descargas de sedimento y nutrientes provenientes tanto de ríos como de desagües urbanos (Herrera, 1990). Esos nutrientes y sedimentos llegan al mar también a través del escurrimiento costero. El objetivo de esta investigación es conocer cómo responde la condición de las comunidades de corales duros a los variados ambientes de estos arrecifes aledaños a asentamientos humanos y propensos igualmente a afectaciones de carácter natural. 


\section{MATERIALES Y MÉTODOS}

\section{Zona de estudio}

El área de estudio incluye el noroeste y noreste de la provincia de $\mathrm{La}$ Habana y parte del litoral de la Ciudad de La Habana (incluida en el norte de esa provincia), entre $\operatorname{los} 23^{\circ} 01^{\prime} \mathrm{LN}$, $82^{\circ} 42^{\prime} \mathrm{LW}$ (este de Bahía de Mariel), y los $23^{\circ} 08^{\prime} \mathrm{LN}, 81^{\circ} 40^{\prime} \mathrm{LE}$ (desembocadura del río Bacunayagua), con una extensión de más de $100 \mathrm{~km}$ a lo largo de la costa (Figura 1).
La costa es rocosa con predominio de formaciones del tipo "seboruco", que incluye segmentos de playas y zonas de desembocadura de ríos. Hacia el centro se encuentra el litoral de la Ciudad de La Habana, con una elevada densidad poblacional y un gran número de drenajes pluviales y vertientes albañales. Las corrientes marinas son fundamentalmente de marea y su dirección oscila de forma inconstante hacia el este y el oeste (Lluis-Riera, 1983).

Figura 1

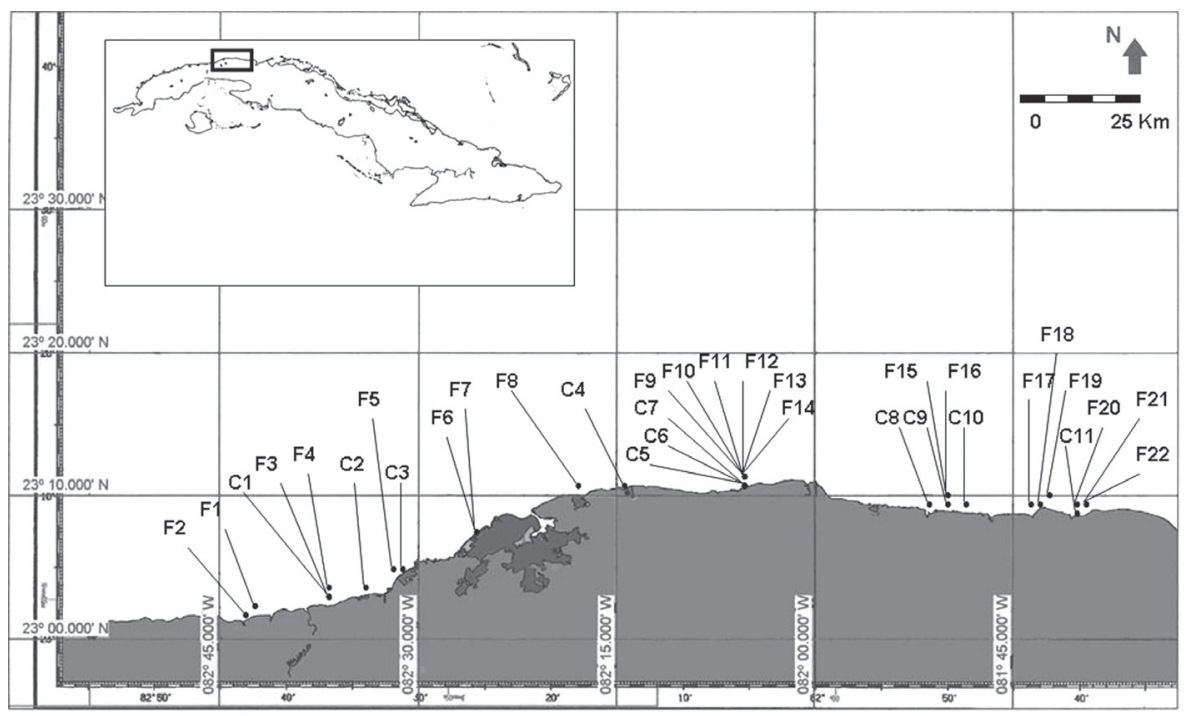

Área de estudio y ubicación de sitios de muestreo. Ver códigos de los sitios en el Cuadro 1. Map with location of sampling sites. Site abbreviations as in the Table 1. 
A lo largo de una estrecha plataforma marina se desarrolla un arrecife del tipo costero con uno o dos niveles de terraza. En algunas localidades cercanas a desembocaduras de ríos aparecen pequeñas crestas arrecifales (de 0.5 a $2 \mathrm{~km}$ de longitud) con desarrollo de Acropora palmata (Lamarck, 1816). A mayor profundidad, el arrecife frontal se presenta continuo con variedad de zonas ecológicas (arrecifes de parche, cañones submarinos o canales, veriles o escarpes y camellones y canales). Las localidades y sitios de trabajo se describen en el Cuadro 1.

\section{Metodología y procesamiento de datos}

La evaluación se realizó entre junio y agosto del 2004. Se escogieron del protocolo AGRRA (Kramer y Lang, 2003) los siguientes métodos de muestreo del bentos:

- Transectos de $10 \mathrm{~m}$ de largo desplegados al azar a lo largo de tramos de aproximadamente $100 \mathrm{~m}$ para: estimar el cubrimiento del fondo por coral duro vivo, conteo y medición del diámetro máximo de los corales interceptados, examinar las enfermedades y la mortalidad reciente y antigua de los corales, referenciar la posición de los marcos cuadrados para conteo de nuevos reclutas de corales y muestrear una banda de $1 \mathrm{~m}$ de ancho para estimar la densidad del erizo negro de espinas largas (Diadema antillarum). Se analizaron un total de 425 unidades de muestreo.

- Marcos cuadrados de $25 \mathrm{~cm}$ de lado para conteo de nuevos reclutas de corales (cinco marcos en cada transecto), ubicados en cinco posiciones a lo largo de cada transecto $(1 \mathrm{~m}, 3 \mathrm{~m}, 5 \mathrm{~m}, 7 \mathrm{~m}$ y 9 $\mathrm{m})$, estimación del cubrimiento del sustrato por césped algal (turf, algas filamentosas menores de $1 \mathrm{~cm}$ de altura) y/o macroalgas carnosas, calcáreas y costrosas.

Para la identificación de los órdenes Scleractinia y Milleporina se siguieron los criterios de Steneck et al. (1997). Se incluyeron solamente las especies y colonias con más de 10 $\mathrm{cm}$ de diámetro máximo. Las algas y los erizos se muestrearon entre junio y agosto.

Se determinó la abundancia y composición por especies de corales. Se utilizaron como índices de diversidad, la riqueza de la especie (número total de especies contadas), la heterogeneidad de Shannon (Shannon y Weaver, 1963) y la equitatividad de Pielou (1966). El tamaño de la muestra se consideró apropiado, dado que las curvas de número de especies contadas contra el número de colonias acumuladas mostraron tendencia asintótica en todos los casos.

Se calcularon la media y el error estándar de los indicadores de cubrimiento vivo de coral, la densidad lineal de corales adultos y densidad 


\section{Cuadro 1}

Sitios de crestas y arrecifes frontales, localización y profundidad.

\section{Table 1}

Location and depth of crest and fore-reefs sites.

\begin{tabular}{|c|c|c|c|c|c|}
\hline Localidad & Sitio & Zona ecológica & Latitud N & Longitud W & Profundidad \\
\hline Henequén & F1 & veril & $23^{\circ} 01^{\prime} 31$ & $82^{\circ} 43^{\prime} 09$ & $12-15 \mathrm{~m}$ \\
\hline Río Mosquito & $\mathrm{F} 2$ & veril & $23^{\circ} 01^{\prime} 38$ & $82^{\circ} 42^{\prime} 40$ & $12-15 \mathrm{~m}$ \\
\hline \multirow[t]{3}{*}{ Playa El Salado } & $\mathrm{C} 1$ & cresta & $23^{\circ} 02^{\prime} 51$ & $82^{\circ} 36^{\prime} 15$ & $1-2 \mathrm{~m}$ \\
\hline & F3 & veril & $23^{\circ} 02^{\prime} 09$ & $82^{\circ} 36^{\prime} 28$ & $12-15 \mathrm{~m}$ \\
\hline & F4 & camellones & $23^{\circ} 02^{\prime} 41$ & $82^{\circ} 36^{\prime} 21$ & $15-17 \mathrm{~m}$ \\
\hline Playa Baracoa & $\mathrm{C} 2$ & cresta & $23^{\circ} 03^{\prime} 19$ & $82^{\circ} 33^{\prime} 16$ & $1-2 \mathrm{~m}$ \\
\hline \multirow[t]{2}{*}{ Playa Santa Fe } & $\mathrm{C} 3$ & cresta & $23^{\circ} 04^{\prime} 56$ & $82^{\circ} 30^{\prime} 45$ & $1-2 \mathrm{~m}$ \\
\hline & F5 & veril & $23^{\circ} 05^{\prime} 03$ & $82^{\circ} 30^{\prime} 52$ & $12-15 \mathrm{~m}$ \\
\hline \multirow[t]{2}{*}{ Litoral Acuario } & F6 & veril & $23^{\circ} 07^{\prime} 12$ & $82^{\circ} 25^{\prime} 74$ & $12-15 \mathrm{~m}$ \\
\hline & F7 & camellones & $23^{\circ} 07^{\prime} 26$ & $82^{\circ} 26^{\prime} 04$ & $15-17 \mathrm{~m}$ \\
\hline Poblado de Cojímar & F8 & veril & $23^{\circ} 10^{\prime} 14$ & $82^{\circ} 17^{\prime} 41$ & $12-15 \mathrm{~m}$ \\
\hline Playa Bacuranao & $\mathrm{C} 4$ & cresta & $23^{\circ} 10^{\prime} 46$ & $82^{\circ} 14^{\prime} 26$ & $1-2 \mathrm{~m}$ \\
\hline \multirow[t]{9}{*}{ Playa Rincón de Guanabo } & $\mathrm{C} 5$ & cresta & $23^{\circ} 10^{\prime} 36$ & $82^{\circ} 06^{\prime} 07$ & $1-2 \mathrm{~m}$ \\
\hline & C6 & cresta & $23^{\circ} 10^{\prime} 35$ & $82^{\circ} 06^{\prime} 55$ & $1-2 \mathrm{~m}$ \\
\hline & $\mathrm{C} 7$ & cresta & $23^{\circ} 10^{\prime} 44$ & $82^{\circ} 06^{\prime} 47$ & $1-2 \mathrm{~m}$ \\
\hline & F9 & veril & $23^{\circ} 11^{\prime} 58$ & $82^{\circ} 06^{\prime} 09$ & $12-15 \mathrm{~m}$ \\
\hline & F10 & veril & $23^{\circ} 11^{\prime} 01$ & $82^{\circ} 06^{\prime} 00$ & $12-15 \mathrm{~m}$ \\
\hline & F11 & veril & $23^{\circ} 11^{\prime} 02$ & $82^{\circ} 05^{\prime} 45$ & $12-15 \mathrm{~m}$ \\
\hline & F12 & camellones & $23^{\circ} 11^{\prime} 08$ & $82^{\circ} 06^{\prime} 14$ & $20 \mathrm{~m}$ \\
\hline & F13 & camellones & $23^{\circ} 11^{\prime} 10$ & $82^{\circ} 05^{\prime} 58$ & $20 \mathrm{~m}$ \\
\hline & F14 & camellones & $23^{\circ} 11^{\prime} 12$ & $82^{\circ} 05^{\prime} 44$ & $20 \mathrm{~m}$ \\
\hline Campismo Peñón del Fraile & $\mathrm{C} 8$ & cresta & $23^{\circ} 09^{\prime} 37$ & $81^{\circ} 51^{\prime} 23$ & $1-2 \mathrm{~m}$ \\
\hline \multirow[t]{3}{*}{ Río Trópico } & C9 & cresta & $23^{\circ} 09^{\prime} 14$ & $81^{\circ} 49^{\prime} 58$ & $1-2 \mathrm{~m}$ \\
\hline & F15 & cabezos & $23^{\circ} 09^{\prime} 27$ & $81^{\circ} 49^{\prime} 50$ & $8-10 \mathrm{~m}$ \\
\hline & F16 & canal & $23^{\circ} 09^{\prime} 27$ & $81^{\circ} 49^{\prime} 61$ & $10 \mathrm{~m}$ \\
\hline Campismo Peñas Blancas & $\mathrm{C} 10$ & cresta & $23^{\circ} 08^{\prime} 56$ & $81^{\circ} 47^{\prime} 37$ & $1-2 \mathrm{~m}$ \\
\hline Palmarejo & F17 & camellones & $23^{\circ} 08^{\prime} 13$ & $81^{\circ} 43^{\prime} 21$ & $15-17 \mathrm{~m}$ \\
\hline \multirow{2}{*}{$\begin{array}{l}\text { Campismo Puerto } \\
\text { Escondido }\end{array}$} & F18 & cabezos & $23^{\circ} 09^{\prime} 28$ & $81^{\circ} 43^{\prime} 21$ & $8-10 \mathrm{~m}$ \\
\hline & F19 & camellones & $23^{\circ} 09^{\prime} 19$ & $81^{\circ} 43^{\prime} 21$ & $15-17 \mathrm{~m}$ \\
\hline \multirow[t]{4}{*}{ Río Bacunayagua } & $\mathrm{C} 11$ & cresta & $23^{\circ} 08^{\prime} 45$ & $81^{\circ} 40^{\prime} 30$ & $2 \mathrm{~m}$ \\
\hline & F20 & cabezos & $23^{\circ} 08^{\prime} 50$ & $81^{\circ} 40^{\prime} 26$ & $8-10 \mathrm{~m}$ \\
\hline & $\mathrm{F} 21$ & veril & $23^{\circ} 08^{\prime} 55$ & $81^{\circ} 40^{\prime} 17$ & $12-15 \mathrm{~m}$ \\
\hline & F22 & camellones & $23^{\circ} 08^{\prime} 59$ & $81^{\circ} 40^{\prime} 12$ & $18 \mathrm{~m}$ \\
\hline
\end{tabular}


de reclutas, el diámetro máximo, la mortalidad antigua y mortalidad reciente de las colonias y la densidad de erizos negros de espinas largas $(D$. antillarum). Se estimaron los porcentajes medios de abundancia relativa de los distintos grupos morfofuncionales de algas. Se calculó el índice de macroalgas carnosas mediante la multiplicación del valor de la altura promedio del dosel de las macroalgas presentes dentro del marco cuadrado por su porcentaje de cubrimiento (Kramer y Lang, 2003).

Para conocer la existencia de diferencias significativas entre sitios se realizó un análisis de varianza unifactorial (ANOVA). Se comprobó la normalidad y homogeneidad de varianza de los datos a partir de la relación propuesta por Taylor (1961), según la metodología de Elliot (1971). Se transformaron los datos según fue necesario. Para determinar las diferencias entre los pares de media se utilizó la prueba de Student-Newman-Keuls, con índice de significación de 0.05 . Todos los cálculos se realizaron ejecutando el programa Statistica 6.0 (StatSoft, Inc., 2002).

\section{RESULTADOS}

En las crestas se encontraron de 7 a 12 especies por estación, la heterogeneidad de Shannon varió entre 1.21 y 2.08 natios y la equitatividad entre 0.6 y 0.8 . Como promedio general del área de estudio, dominaron numéricamente Millepora compla- nata (Linnaeus, 1758) y A. palmata, conformando el $40 \%$ del total de colonias contadas. El mayor predominio de ambas se observó en los sitios situados hacia el oeste del área estudiada y en Bacunayagua (Cuadro 2).

En los arrecifes frontales, la riqueza de especies varió entre 12 y 22; la heterogeneidad de Shannon, entre 1.82 y 2.43 natios y la equitatividad, entre 0.73 y 0.88 . Según el promedio general del área de estudio, predominaron en orden de abundancia: Agaricia agaricites (Linnaeus, 1758), Montastraea cavernosa (Linnaeus, 1767), Montastraea faveolata (Ellis y Solander, 1786), Siderastrea siderea (Ellis y Solander, 1786) y Porites astreoides (Lamarck, 1816). Estas constituyeron, en conjunto, el $71 \%$ del total de colonias contadas en todos los sitios (Cuadro 3).

El cubrimiento de coral vivo en las crestas varió del 10 al 40\%. En Santa Fe y Bacuranao fue significativamente inferior, mientras que en el resto de los sitios fue mayor a $25 \%$. La densidad de colonias varió entre 5 y 12 colonias $/ 10 \mathrm{~m}$. Se destacaron por sus densidades más altas el este del Rincón de Guanabo, el oeste del Trópico, Peñas Blancas y Bacunayagua. La talla promedio de las colonias varió de 60 a $160 \mathrm{~cm}$ y los valores más altos se registraron en Trópico y Bacunayagua. El reclutamiento no mostró diferencias entre sitios, con una media menor que $1 \mathrm{recluta} / \mathrm{m}^{2}$ (Figura 2). 


\section{Cuadro 2}

Abundancia (\%) y lista de especies de corales en crestas que conforman el $90 \%$ del total de colonias contadas, e índices de diversidad. $\mathrm{N}=$ número de colonias, $\mathrm{S}=$ riqueza de especies, $\mathrm{H}^{\prime}=$ heterogeneidad de Shannon, $\mathrm{J}^{\prime}=$ Equitatividad de Pielou. Ver códigos de los sitios en el Cuadro 1.

\section{Table 2}

Abundance (\%) and list of species of stony corals in crests that conform $90 \%$ of the total of counted colonies, and indexes of diversity. $\mathrm{N}=$ number of colonies, $\mathrm{S}=$ number of species, $\mathrm{H}^{\prime}=$ Shannon heterogeneity, $\mathrm{J}^{\prime}=$ Pielou evenness. To see codes of the sites in the Table 1 .

\begin{tabular}{lrrrrrrrrrrrr}
\hline $\begin{array}{c}\text { Especies e índices de } \\
\text { diversidad }\end{array}$ & \multicolumn{10}{c}{ Sitios } \\
\cline { 2 - 11 } & $\mathbf{C 1}$ & $\mathbf{C 2}$ & $\mathbf{C 3}$ & $\mathbf{C 4}$ & $\mathbf{C 5}$ & $\mathbf{C 6}$ & $\mathbf{C 7}$ & $\mathbf{C 8}$ & $\mathbf{C 9}$ & $\mathbf{C 1 0}$ & $\mathbf{C 1 1}$ \\
\hline Millepora complanata & 48.0 & 31.6 & 24.7 & 8.5 & 25.0 & 50.0 & 28.9 & 10.8 & 21.1 & 17.1 & 39.8 \\
Acropora palmata & 31.1 & 44.5 & 37.1 & 27.0 & 58.9 & 30.9 & 41.2 & 12.6 & 19.1 & 26.5 & 39.8 \\
Porites astreoides & 9.5 & 7.7 & 4.1 & 31.9 & 7.1 & 7.3 & 5.3 & 20.7 & 20.1 & 2.6 & 3.9 \\
Acropora cervicornis & 0.0 & 0.0 & 0.0 & 0.0 & 2.7 & 5.5 & 5.3 & 0.9 & 22.2 & 26.5 & 0.0 \\
Agaricia agaricites & 0.0 & 0.0 & 13.4 & 2.1 & 0.0 & 2.7 & 4.4 & 28.8 & 7.2 & 0.9 & 0.0 \\
Acropora prolifera & 0.0 & 0.0 & 0.0 & 0.0 & 0.0 & 0.0 & 0.0 & 0.0 & 0.0 & 17.1 & 0.0 \\
Diploria clivosa & 3.4 & 6.5 & 7.2 & 6.4 & 1.8 & 0.0 & 1.8 & 1.8 & 1.5 & 1.7 & 0.0 \\
\hline S (especies) & 9 & 8 & 11 & 11 & 9 & 8 & 12 & 14 & 11 & 12 & 7 \\
H' (natios) & 1.4 & 1.4 & 1.8 & 1.9 & 1.2 & 1.3 & 1.7 & 2.1 & 1.9 & 1.8 & 1.3 \\
J' & 0.6 & 0.7 & 0.7 & 0.8 & 0.6 & 0.6 & 0.7 & 0.8 & 0.8 & 0.7 & 0.7 \\
\hline N & 148 & 155 & 97 & 141 & 112 & 110 & 114 & 111 & 194 & 117 & 103 \\
\hline
\end{tabular}




\section{Cuadro 3}

Abundancia (\%) y lista de especies de corales en arrecifes frontales que conforman el $90 \%$ del total de colonias contadas, e índices de diversidad. $\mathrm{N}=$ total de colonias, $\mathrm{S}=$ riqueza de especies, $\mathrm{H}^{\prime}=$ heterogeneidad de Shannon y Weaver, J' = equitatividad de Pielou. Ver códigos de los sitios en el Cuadro 1.

\section{Table 3}

Abundance (\%) and list of species of stony corals in fore-reefs that conform $90 \%$ of the total of counted colonies, and indexes of diversity. $\mathrm{N}=$ number of colonies, $\mathrm{S}=$ number of species, $\mathrm{H}^{\prime}=$ Shannon heterogeneity, J'=Pielou evenness. To see codes of the sites in the Table 1.

\begin{tabular}{|c|c|c|c|c|c|c|c|c|c|c|c|}
\hline \multirow{2}{*}{$\begin{array}{c}\text { Especies e índices de } \\
\text { diversidad }\end{array}$} & \multicolumn{11}{|c|}{ Sitios } \\
\hline & F1 & F2 & F3 & F4 & F5 & F6 & F7 & F8 & F9 & F10 & F11 \\
\hline Agaricia agaricites & 28.1 & 20.7 & 15.8 & 33.3 & 18.1 & 7.4 & 21.6 & 18.8 & 12.4 & 17.9 & 16.7 \\
\hline Montastraea cavernosa & 9.4 & 27.6 & 22.3 & 2.0 & 27.1 & 26.3 & 8.8 & 18.8 & 12.4 & 10.3 & 7.1 \\
\hline Montastraea faveolata & 2.3 & 5.2 & 24.5 & 30.7 & 13.0 & 4.2 & 7.8 & 0.0 & 13.3 & 21.4 & 21.4 \\
\hline Siderastraea siderea & 13.3 & 12.1 & 7.6 & 5.2 & 9.0 & 17.9 & 11.8 & 37.5 & 19.0 & 8.5 & 6.3 \\
\hline Porites astreoides & 5.5 & 2.6 & 6.5 & 9.8 & 10.2 & 13.7 & 17.6 & 8.3 & 3.8 & 5.1 & 9.5 \\
\hline Porites porites & 17.2 & 2.6 & 1.1 & 2.0 & 1.1 & 6.3 & 2.0 & 0.0 & 14.3 & 17.9 & 21.4 \\
\hline Montastraea franksi & 0.0 & 0.9 & 4.9 & 21.6 & 0.6 & 0.0 & 4.9 & 0.0 & 1.9 & 1.7 & 9.5 \\
\hline Madracis decactis & 3.9 & 2.6 & 3.8 & 0.0 & 4.0 & 6.3 & 5.9 & 2.1 & 6.7 & 2.6 & 1.6 \\
\hline Meandrina meandrites & 5.5 & 5.2 & 0.5 & 0.7 & 6.2 & 3.2 & 2.0 & 3.1 & 1.9 & 2.6 & 0.8 \\
\hline Millepora alcycornis & 1.6 & 3.4 & 0.5 & 1.3 & 1.7 & 3.2 & 5.9 & 0.0 & 4.8 & 0.0 & 0.8 \\
\hline Diploria strigosa & 1.6 & 1.7 & 3.3 & 2.0 & 1.7 & 2.1 & 0.0 & 0.0 & 2.9 & 1.7 & 2.4 \\
\hline $\mathbf{S}$ (especies) & 16 & 18 & 21 & 12 & 18 & 13 & 14 & 12 & 16 & 16 & 17 \\
\hline $\mathbf{H}^{\prime}$ (natios) & 2.26 & 2.25 & 2.29 & 2.02 & 2.22 & 2.21 & 2.33 & 1.82 & 2.36 & 2.27 & 2.25 \\
\hline $\mathbf{J}^{\prime}$ & 0.82 & 0.78 & 0.75 & 0.81 & 0.77 & 0.86 & 0.88 & 0.73 & 0.85 & 0.82 & 0.79 \\
\hline $\mathbf{N}$ & 128 & 116 & 184 & 122 & 177 & 95 & 102 & 96 & 105 & 117 & 126 \\
\hline
\end{tabular}


Cuadro 3 (Continuación)

Table 3 (Continuation)

\begin{tabular}{|c|c|c|c|c|c|c|c|c|c|c|c|}
\hline \multirow{2}{*}{$\begin{array}{l}\text { Especies e índices de } \\
\text { diversidad }\end{array}$} & \multicolumn{11}{|c|}{ Sitios } \\
\hline & F12 & F13 & F14 & F15 & F16 & F17 & F18 & F19 & F20 & F21 & F22 \\
\hline Agaricia agaricites & 19.4 & 14.0 & 17.0 & 31.3 & 25.9 & 8.6 & 16.3 & 9.4 & 19.5 & 14.0 & 29.9 \\
\hline Montastraea cavernosa & 17.2 & 16.0 & 16.0 & 8.9 & 31.0 & 6.2 & 10.3 & 13.2 & 8.0 & 32.0 & 23.7 \\
\hline Montastraea faveolata & 12.9 & 8.0 & 10.6 & 19.2 & 10.3 & 32.1 & 21.7 & 11.3 & 23.0 & 9.9 & 4.1 \\
\hline Siderastraea siderea & 19.4 & 15.0 & 13.8 & 10.3 & 6.9 & 16.0 & 5.9 & 17.0 & 6.9 & 14.0 & 8.2 \\
\hline Porites astreoides & 7.5 & 11.0 & 9.6 & 3.7 & 7.8 & 6.2 & 7.4 & 9.4 & 8.0 & 2.9 & 8.2 \\
\hline Porites porites & 0.0 & 0.0 & 0.0 & 2.8 & 0.0 & 7.4 & 13.3 & 1.9 & 5.7 & 0.0 & 6.2 \\
\hline Montastraea franksi & 8.6 & 2.0 & 3.2 & 1.4 & 0.9 & 2.5 & 1.5 & 11.3 & 2.9 & 1.7 & 0.0 \\
\hline Madracis decactis & 3.2 & 5.0 & 3.2 & 1.4 & 3.4 & 2.5 & 0.0 & 1.9 & 2.3 & 0.6 & 3.1 \\
\hline Meandrina meandrites & 1.1 & 3.0 & 4.3 & 1.9 & 0.0 & 1.2 & 0.5 & 7.5 & 1.1 & 7.0 & 5.2 \\
\hline Millepora alcycornis & 5.4 & 7.0 & 6.4 & 1.4 & 0.9 & 2.5 & 3.4 & 13.2 & 1.1 & 0.6 & 1.0 \\
\hline Diploria strigosa & 0.0 & 0.0 & 0.0 & 1.4 & 2.6 & 7.4 & 2.0 & 3.8 & 6.3 & 2.3 & 3.1 \\
\hline $\mathbf{S}$ (especies) & 14 & 14 & 15 & 22 & 13 & 15 & 21 & 12 & 17 & 15 & 14 \\
\hline $\mathbf{H}^{\prime}$ (natios) & 2.22 & 2.41 & 2.41 & 2.32 & 1.97 & 2.22 & 2.43 & 2.32 & 2.36 & 2.14 & 2.11 \\
\hline $\mathrm{J}$ ' & 0.84 & 0.91 & 0.89 & 0.75 & 0.77 & 0.82 & 0.80 & 0.93 & 0.83 & 0.79 & 0.80 \\
\hline $\mathbf{N}$ & 93 & 100 & 94 & 214 & 116 & 81 & 203 & 53 & 174 & 172 & 97 \\
\hline
\end{tabular}


Figura 2
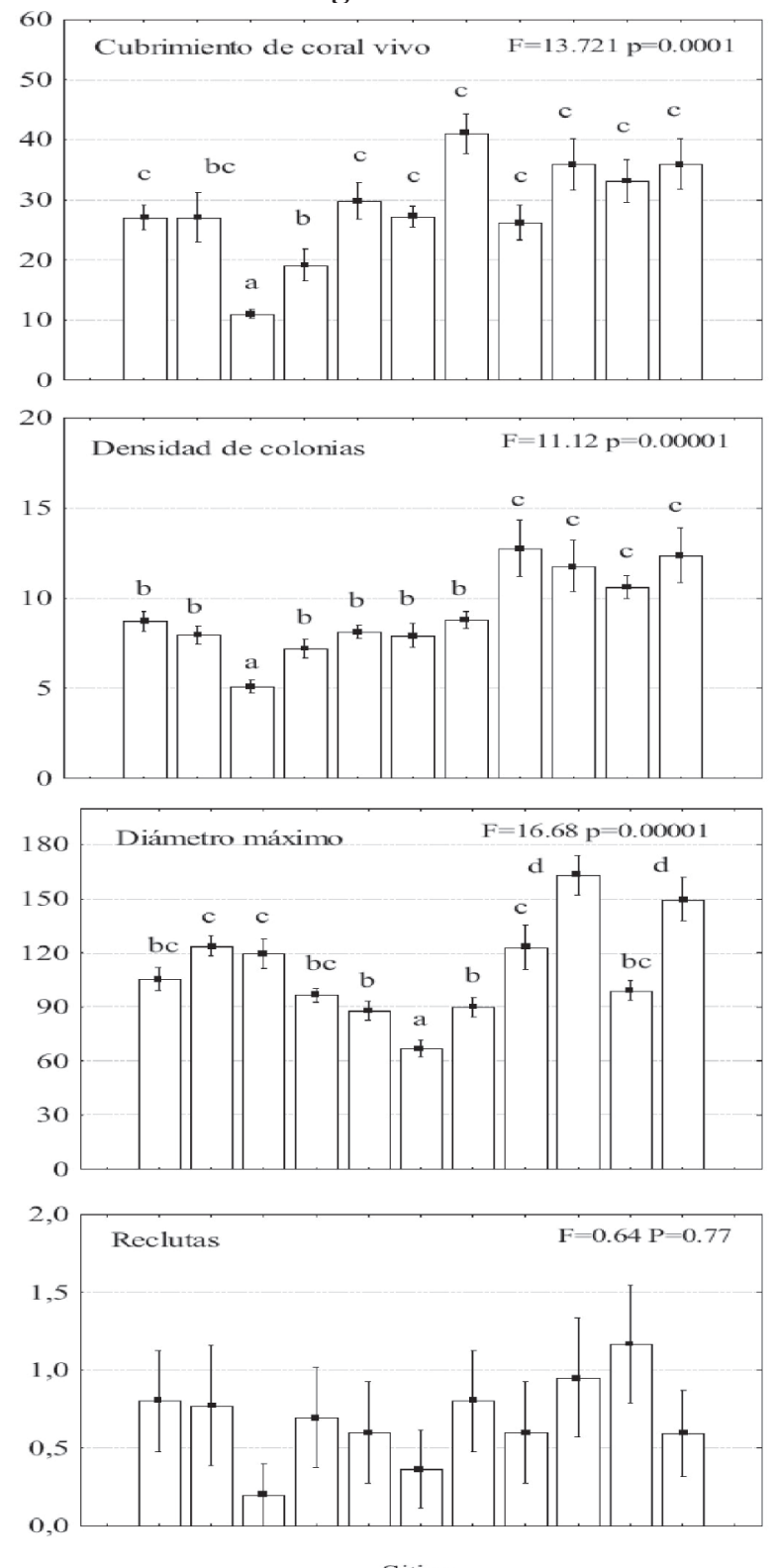

Sitios

Indicadores biológicos (media+error estándar) en los sitios de crestas. Las letras diferentes indican variación significativa. Ver Cuadro 1 para los códigos de cada sitio.

Biological indicators at the crest sites (mean+standar error). Different letters indicate significant differences. Site abbreviations as in the Table 1. 
El promedio de cubrimiento de coral vivo en los arrecifes frontales fue de $21.4 \%$. Se encontró variabilidad entre sitios donde los valores medios inferiores rodearon el $10 \%$ y los superiores llegaron a alcanzar un $40 \%$. Los tres sitios de cabezos (Trópico, Puerto Escondido y Bacunayagua) y el veril de Bacunayagua se destacaron por presentar cubrimientos mayores a $30 \%$. La densidad promedio de colonias fue de 12.1 colonias $/ 10 \mathrm{~m}$, con gran variación entre sitios. En los cabezos y el veril de Bacunayagua se llegó a contar más de 20 colonias $/ 10 \mathrm{~m}$. El diámetro promedio de las colonias varió de 20 a $45 \mathrm{~cm}$. En el canal del Trópico y en los sitios de cabezos se encontraron las mayores tallas promedio. La densidad de reclutas mostró variación significativa entre sitios y sus promedios variaron de 7 a 11 colonias $/ \mathrm{m}^{2}$ (Figura 3).

En las crestas, los promedios de mortalidad antigua variaron entre 9 y $62 \%$, registrándose el mayor porcentaje en Santa Fe. La mortalidad reciente fue baja, con un máximo de $3.5 \%$ en Bacunayagua (Figura 4). En los arrecifes frontales, la mortalidad reciente fue menor que en las crestas, con no más de $2.5 \%$. La mortalidad antigua varió de 5 a $19 \%$, con un promedio de 12\% (Figura 5).

Las enfermedades y los organismos bioerodadores fueron los principales causantes de la escasa mortalidad reciente en los corales duros. En las crestas se encontraron "manchas blancas" sobre A. palmata y A. cervicornis (Lamarck, 1816), atribuibles a depredación por el molusco Coralliophilla sp., dada su presencia física sobre los corales afectados. Se encontró "plaga blanca" en cinco arrecifes frontales, con la mayor cantidad de colonias enfermas en río Mosquito. Las especies agredidas por esta enfermedad fueron: Dichocoenia stockesi (Milne Edwards y Haime, 1848), $A$. agaricites, Stephanocoenia intersepta (Esper, 1795) y Leptoseris cucullata (Cairns, 1982). Se encontró igualmente el síndrome de "manchas oscuras" sobre Siderastrea siderea en 8 sitios.

En las crestas, el césped algal promedió un $38 \%$, las algas carnosas un $28 \%$, las algas costrosas un $18 \%$ y las algas calcáreas un $15 \%$. La densidad promedio de $D$. antillarum fue de 40 individuos $/ 100 \mathrm{~m}^{2}$ (Figura 6).

En los arrecifes frontales, las algas carnosas fueron las más abundantes y mostraron un cubrimiento promedio de $40 \%$. El césped algal registró un promedio de $35 \%$, las algas calcáreas un $12 \%$ y las algas costrosas un $10 \%$. La densidad promedio de $D$. antillarum estuvo por debajo de 20 individuos $/ 100 \mathrm{~m}^{2}$ (Figura 6).

La media del índice de macroalgas carnosas en las crestas fue de 55,6 . Por debajo de esta se destacaron El Salado y Peñas Blancas, con valores cercanos a 20. En arrecifes frontales, este índice promedió 130, alcanzando en Santa Fe, Litoral Acuario y Cojímar registros superiores a 200 (Figura 7). 
Figura 3
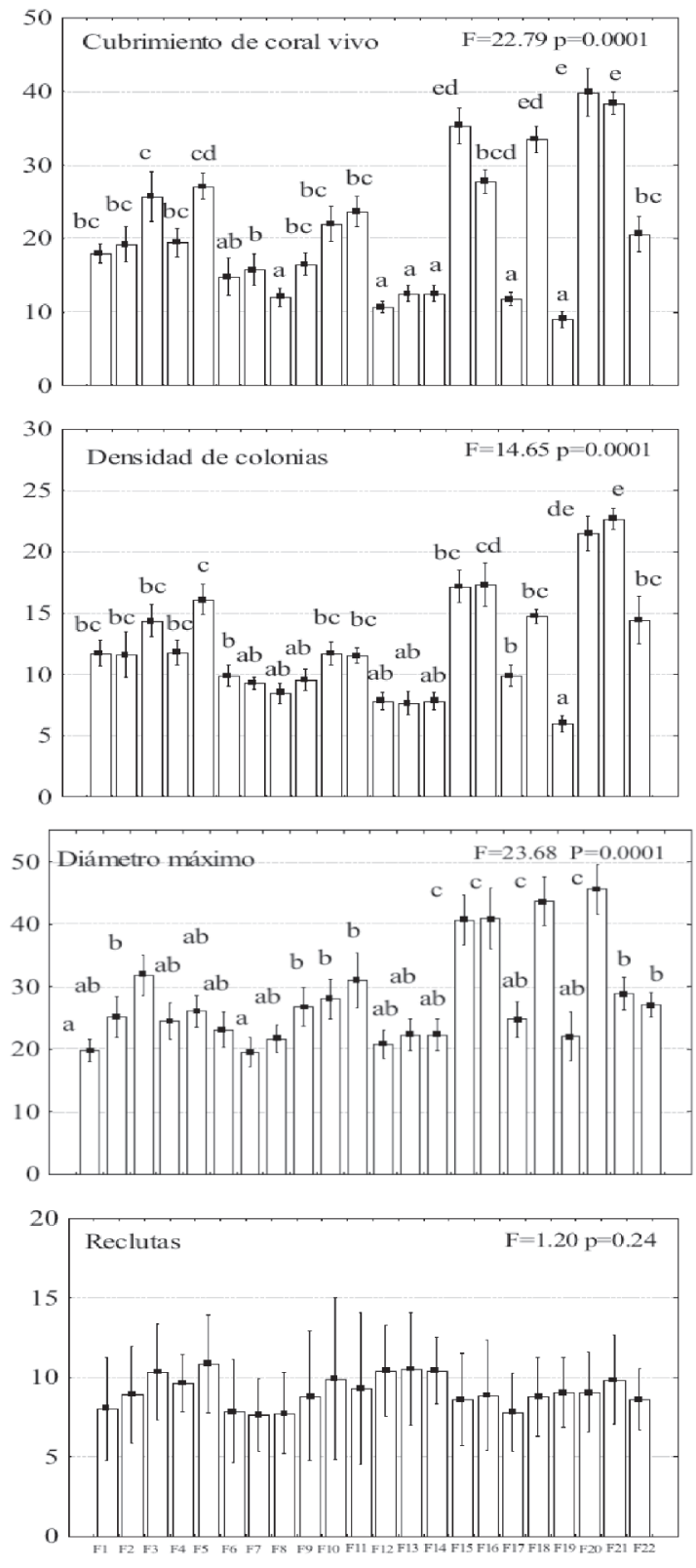

Sitios

Indicadores biológicos en sitios de arrecifes frontales. Las letras diferentes indican variación significativa.Ver Cuadro 1 para los códigos de cada sitio.

Biological indicators at the of fore-reefs sites. Different letters indicate significant differences. Site abbreviations as in the Table 1. 
Figura 4
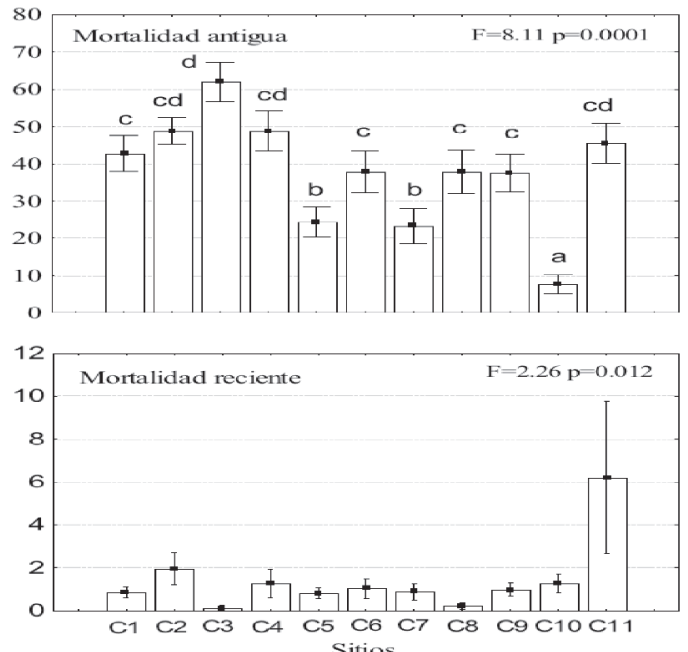

Porcentajes de mortalidad antigua y reciente en crestas. Las letras diferentes indican variación significativa. Ver Cuadro 1 para los códigos de cada sitio.

Percentages of old and recent mortality in crests. Different letters indicate significant differences. Site abbreviations as in the Table 1.

Figura 5
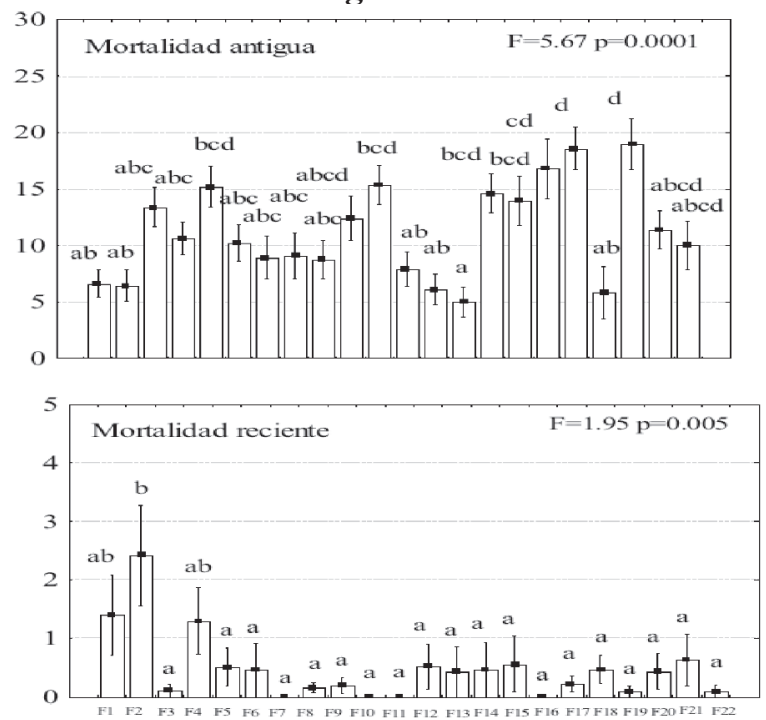

Sitios

Porcentajes de mortalidad antigua y reciente en arrecifes frontales. Las letras diferentes indican variación significativa. Ver Cuadro 1 para los códigos de cada sitio.

Percentages of old and recent mortality in fore-reefs. Different letters indicate significant differences. Site abbreviations as in the Table 1. 
Figura 6

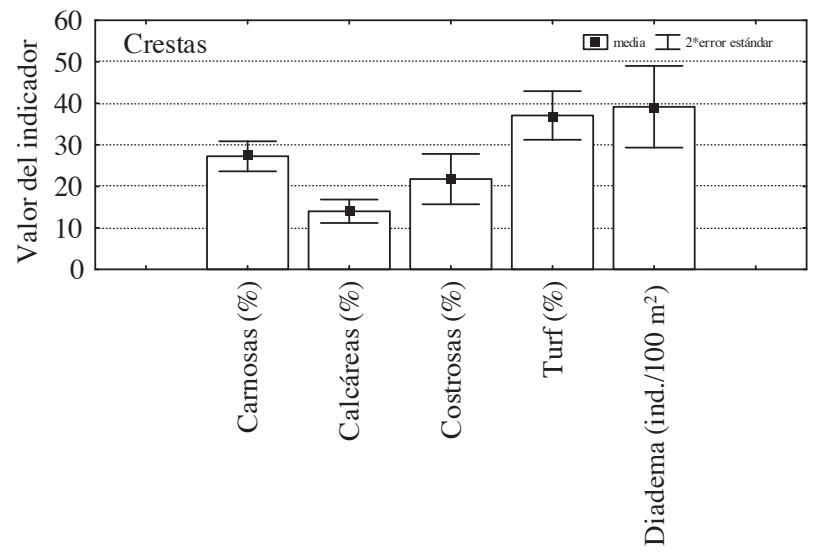

Indicadores

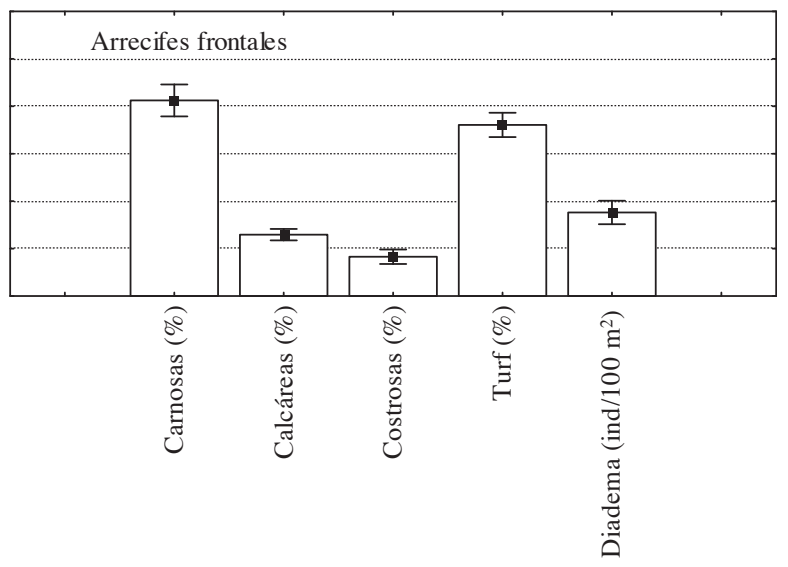

Indicadores

Indicadores de grupos morfofuncionales de algas y densidad de erizos negros para la zona de estudio.

Indicators of algae functional groups and density of black sea-urchins for the study area. Site abbreviations as in the Table 1 . 
Figura 7

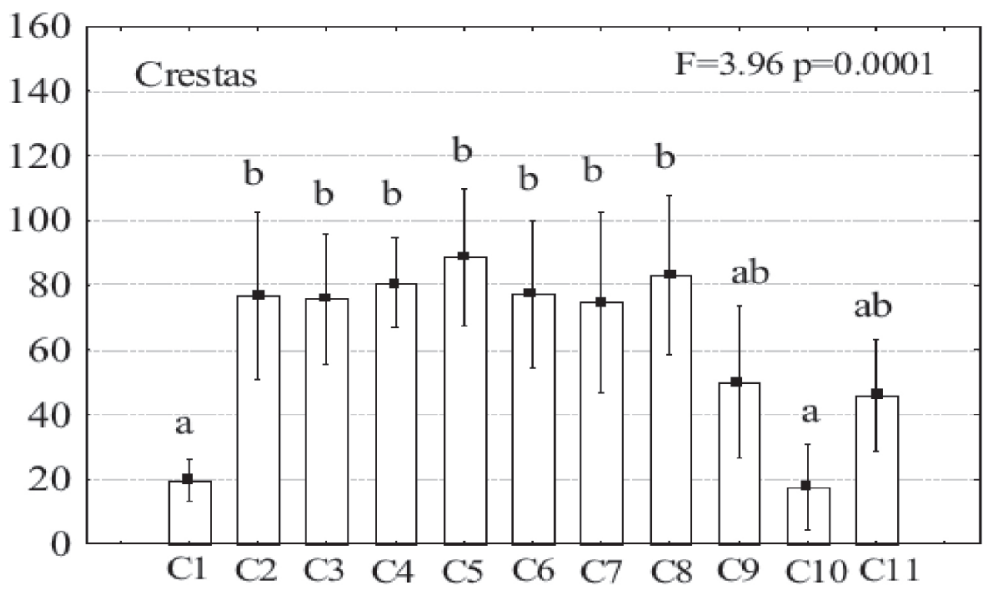

Sitios

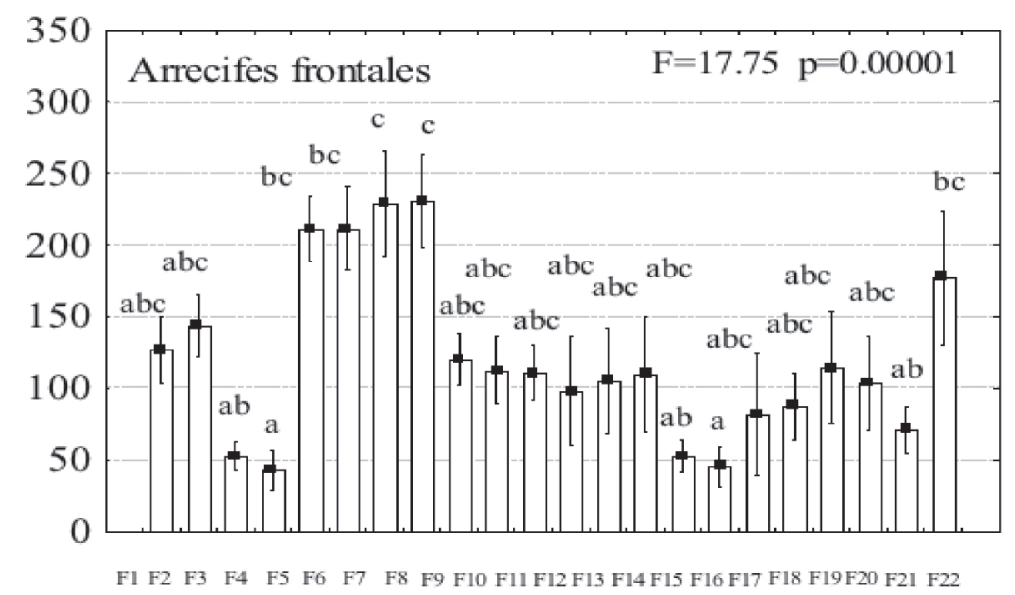

Sitios

Índice de macroalgas carnosas para cada sitio. Las letras diferentes indican variación significativa. En el caso de las crestas, la prueba de comparación de medias (SNK) no pudo determinar diferencias.Ver Cuadro 1 para los códigos de cada sitio.

Fleshy algae index of for each site. Different letters indicate significant differences. In the case of crests, the poc-hoc test (SNK) could not determine differences. Site abbreviations as in the Table 1. 


\section{DISCUSIÓN}

El total de especies identificadas en la zona de estudio comprende el $62.7 \%$ del total de especies reportadas para Cuba, según la lista de corales duros de González-Ferrer et al. (2004). La riqueza de especies por sitios se mantuvo dentro de los márgenes previamente reportados para arrecifes considerados como más limpios de La Habana (Martínez-Estalella y Herrera, 1989; Herrera, 1990; Guardia y González-Sansón, 1997; González, 1999; González-Ferrer, 2000; Caballero y Guardia, 2003; Valdivia y Guardia, 2004; Caballero et al. 2005).

La dominancia de $A$. palmata y M. complanata influyó directamente en la variación del índice de heterogeneidad entre los sitios de crestas. Las crestas de Guanabo, Trópico y Peñas Blancas presentaron el mejor desarrollo estructural propiciado por la primera especie. La existencia de su relieve de cierta zonación hacia la zona trasera ha permitido que especies menos resistentes al oleaje, como A. cervicornis, se establezcan de forma destacada al resguardo de colonias grandes de $A$. palmata.

Los índices de cubrimiento de coral vivo de los arrecifes someros fueron notables e indican condiciones favorables si se comparan con los registrados en esta década para otros arrecifes de Cuba. En crestas del oeste de Cayo Largo, solamente una de las siete evaluadas presentó un cubrimiento de coral vivo por encima del $20 \%$ (Alcolado et al. 2001a). Al sur y al este del Golfo de Batabanó únicamente el $35 \%$ de las crestas presentaron una cubierta viva de coral mayor a $25 \%$ (Alcolado et al. 2001b), mientras que en el archipiélago Jardines de la Reina únicamente lo hizo el 12\% (Alcolado et al. 2001c). En el archipiélago Sabana-Camagüey, en la actualidad, la mayor parte de las crestas se han convertido en bajos rocosos, donde predominan mayoritariamente Millepora, Porites o Diploria, en vez de A. palmata (González-Ferrer et al. 2007a). Todos estos sitios mencionados constituyen arrecifes limpios alejados de fuentes contaminantes y establecimientos poblacionales que se han estado degradando debido a factores asociados al clima.

Las crestas más saludables (con menor porcentaje de mortalidad) fueron las del Rincón de Guanabo, Peñas Blancas y Trópico, áreas comparativamente limpias, donde al parecer los aportes terrígenos no influyen tan negativamente sobre estas. En casi todas las crestas se observó el proceso de recape ("re-sheeting") en colonias de A. palmata que experimentaron mortalidad, lo que crea expectativas de una recuperación más rápida de estas, ya que no se necesita que el coral se desarrolle desde un tamaño pequeño, sino que se aprovechan las grandes estructuras esqueléticas muertas (Alcolado et al. 2001a; Acropora Biological Review Team, 2005). 
La composición por especies en los arrecifes frontales se mostró variable y no indica de manera clara una posible acción notable de factores determinísticos (tensión hidrodinámica, sedimentación, nutrientes). La zona en general debe verse regulada por una acción combinada de variables físicas y efectos estocásticos no posibles de dilucidar, que actúan de forma favorable o negativa en el éxito reproductivo, fijación de las larvas y desarrollo de los corales de cada localidad. Las especies dominantes no muestran una característica particular en cuanto a tolerancia o sensibilidad a cierta fuente de estrés, como para poder inferir algún posible factor selectivo sobre la comunidad.

Los sitios donde los indicadores biológicos reflejaron condiciones más favorables fueron las tres zonas de cabezos (Trópico, Puerto Escondido y Bacunayagua). Esto coincide con lo encontrado en fondos de cabezos igualmente someros de Bahía de Cochinos (sur central de Cuba), área protegida (Parque Nacional Península de Zapata), limpia y con arrecifes saludables, geográficamente resguardada de la acción de los vientos alisios predominantes y los del norte durante la temporada invernal (Caballero et al. 2004). Los porcentajes de cubrimiento vivo de coral en estos cabezos se encuentran por encima de las medias registradas en los últimos diez años para la mayor parte de los arrecifes de Cuba (Alcolado et al. 2001b y c; Guardia et al. 2004 y
2005; Caballero et al. 2007; González-Ferrer et al. 2007a y b).

Los arrecifes de parche o cabezos son estructuras que deben dejar escurrir muy fácilmente los sedimentos por su forma levantada y los surcos que aparecen entre corales vecinos. Estas estructuras del relieve han de haber rebasado con su altura la etapa crítica de amenaza de la fuerte suspensión de los sedimentos del fondo por el oleaje y ya tienen "vía libre" para seguir desarrollándose con la adición de nuevas colonias y/o el crecimiento de las ya existentes. Su estructura física, combinada con una buena iluminación (además de la existencia en el fondo de una capa de arena blanca, donde la reflexión de la luz sobre esta puede brindar a los corales iluminación adicional desde abajo) y un aparente suministro de materia orgánica proveniente de los ríos y de la escorrentía en cantidad admisible por los corales, sugiere condiciones ambientales idóneas para el establecimiento y desarrollo de una gran variedad de especies con disímiles requerimientos ecológicos.

Los sitios donde los indicadores biológicos sugieren condiciones menos favorables fueron los veriles del litoral del Acuario Nacional y Cojímar, los que están en áreas urbanas más pobladas, por lo que es de esperar que estén más afectadas por sedimentos terrígenos y contaminantes. Esto se evidencia en el predominio de $S$. siderea y M. cavernosa (especies resistentes a la sedimentación y 
nutrificación, según Torres y Morelock, 2002), unido a bajos índices de heterogeneidad de especies, de cubrimiento vivo de coral, de densidad y de las tallas de las colonias.

Los índices medios de mortalidad antigua encontrados en los arrecifes frontales no sobrepasan el $22 \%$, que es el promedio del Atlántico Occidental, según las evaluaciones realizadas con el protocolo AGRRA en la región (Kramer, 2003). Los porcentajes de mortalidad reciente indican la no ocurrencia de ningún evento negativo reciente de consideración previo a la presente evaluación. $\mathrm{La}$ ocurrencia de enfermedades tampoco fue de importancia, aunque es necesario dar un seguimiento al grado de incidencia de estas, dada la conducta estacional o temporal de algunas (Green y Bruckner, 2000).

La especie que ha tenido mayor mortalidad tanto en arrecifes someros como profundos fue A. cervicornis (en especial en los biotopos de cabezos y en la zona trasera de la cresta del Rincón de Guanabo). No fue posible cuantificar con precisión el porcentaje de mortalidad de esta especie, ya que a diferencia de los corales masivos, no suele mantener tan íntegro su esqueleto al morir. Sin embargo, en varios sitios sí se observaron parches con restos de colonias muertas con evidencias de marcado desarrollo de estas con anterioridad. Se ha reportado que esta especie ha sufrido un gran deterioro en el Gran Caribe y es considerada como sensible a los cambios de salinidad, temperatura, radiación y tensión hidrodinámica (Brown, 1997; Muller-Parker y D'Elia, 1997; Acropora Biological Review Team, 2005). Alcolado et al. (2001a) infirieron a partir de comparaciones con publicaciones de D. H. H. Kuhlmann, una virtual desaparición de conglomerados de esta especie en Cayo Largo del Sur y desconocen si la mortalidad de esta haya sido causada por blanqueamiento, microorganismos, ciclones o la acción conjunta de algunos de estos factores.

Las densidades de reclutamiento de coral no fueron altas, aunque por lo general el reclutamiento de juveniles en arrecifes del Gran Caribe es limitado aun siendo las condiciones favorables, lo que se debe en gran parte a la biología reproductiva y larval de las principales especies constructoras de arrecifes (Guzmán y Guevara, 1998). En el área se aprecian dos factores que influyen de forma negativa en el reclutamiento: la acción constante y erosiva de los frentes fríos, y el alto cubrimiento del sustrato por macroalgas carnosas que compiten por el espacio con los reclutas y afectan directamente a estos y a los corales jóvenes (Richmond, 1997). Los reclutas que predominaron fueron de A. agaricites, $P$. astreoides y $S$. siderea. Las dos primeras son especies incubadoras y generalmente experimentan mayor reclutamiento dadas sus características de estrategas r (Bak et al. 1977; 
Bak y Steward, 1980). S. siderea es una especie desovadora, sin embargo, sus reclutas se caracterizan por presentar gran resistencia en sus primeras etapas (Bak et al. 1977; Bak y Steward, 1980).

Los criterios de Lapointe et al. (1997) sobre los índices de macroalgas carnosas en un arrecife sugieren que en el área de estudio existen aportes considerables de nutrientes unidos a un insuficiente nivel de herbivorismo. En las crestas, los indicadores de macroalgas carnosas fueron menores; sin embargo, el crecimiento de estas generalmente es controlado por el oleaje, además de por el erizo negro de espinas largas. Dominaron en muchos sitios las dictiotáceas y Lobophora variegata, las cuales algunos autores consideran oportunistas y dominantes en lugares con altos niveles de nutrientes (Delgado y Lapointe, 1994; Lapointe, 1997). Sin embargo, y de modo contradictorio a estos, un artículo más reciente (McClanahan, 2004) plantea que las algas verdaderamente indicadoras de nutrificación en arrecifes de coral son las verdes filamentosas y foliosas, las cuales no se encontraron como dominantes en ningún sitio muestreado en esta evaluación.

A manera de conclusión, se puede plantear que es interesante y parece paradójico que esos arrecifes cercanos a asentamientos humanos, estén en mejores condiciones que arrecifes en lugares con escasa intervención humana, como los de los archipiélagos de los Canarreos y Jardines de la Reina (anteriormente citados en el análisis de los registros de cubrimiento vivo de coral). Esto podría estar dado por cierto nivel de heterotrofía favorecido por la productividad del agua por enriquecimiento y por materia orgánica particulada, ambos provenientes de la tierra. Una mayor productividad del agua contribuye a la heterotrofía en los corales como fuente alternativa o complementaria de alimentación aparte autotrófica a través de las zooxantelas (Schuttenberg y Marshal, 2008). Eso ayudaría a los corales a resistir ante procesos adversos como los eventos de blanqueamiento, y además favorece una recuperación más rápida de cualquier afectación.

\section{BIBLIOGRAFÍA}

Acropora Biological Review Team (2005). Atlantic Acropora Status Review Document. Report to National Marine Fisheries Service, Southeast Regional Office, 3.

Alcolado, P. M., Claro, R., Menéndez,

G. and Martínez-Daranas, B. (1997). General status of Cuban Coral Reefs. Proc. 8th Int. Sym. Coral Reefs, 1, 341-344.

Alcolado, P. M., Claro-Madruga, R. and Estrada, R. (2000a). Status and Prospective of Coral Reef Management in Cuba. Coral Reef Status Report for Cuba, GCRMN. Recuperado en 
diciembre 14, 2007, disponible en http://www.reefbase.org/pdf/ $\% 20$ GCRMN2000CUB.pdf.

Alcolado, P. M., Claro, R., MartínezDaranas, B., Menéndez-Macías, G., García-Parrado, P., Cantelar, K., Espinosa, J., del Valle, R., Martínez, J. C., Neff, T., Hernández-González, M., Hernández-López, J. L. y García-Díaz, T. (2000b). Estado general de los arrecifes coralinos de Cuba y propuestas de manejo ambiental: Informe Final. Instituto de Oceanología de Cuba.

Alcolado, P. M., Claro-Madruga, R., Martínez-Daranas, B., MenéndezMacía, G., García-Parrado, P., Cantelar, K., Hernández, M. y del Valle, R. (2001a). Evaluación ecológica de los arrecifes coralinos del oeste de Cayo Largo del Sur, Cuba: 1998-1999. Bol. Mar. Coas. Res., 30, 25-32.

Alcolado, P. M., Ginsburg, R. N., Kramer, P., Kosminin, V., de la Guardia, E., González, S. y Hernández, M. (2001b). Estado de salud del bentos de los arrecifes coralinos del sur y este del Golfo de Batabanó: Informe Preliminar de la Expedición CUBAGRRA, marzo/2001 (Informefinal). Archivo del Instituto de Oceanología.

Alcolado, P. M., Ginsburg, R., Lang, J. C., Kramer, P. A., Marks, K., Rodríguez, E. A., Andre-Fouet, F., Zlatarski,V., Guardia, E., Pina, F., Cantelar, K., González, S., Caballero, H., Hernández, M. y Hernández, J. L. (2001c). Estado de salud de los arrecifes coralinos del Archipiélago Jardines de la Reina (SE de Cuba): Informe Preliminar de la Expedición CUBAGRRA II. Instituto de Oceanología de Cuba.

Bak, R.P.M., Brouns, J.J.W.M. and Heys, F.M.L. (1977). Regeneration and aspects of spatial competition in the scleractinian corals Agaricia agaricites and Montastraea annularis. Proc. 3th Int. Sym. Coral Reefs, 1, 143-148.

Bak, R.P.M. and Steward-van, E. (1980). Regeneration of superficial damage in the scleraactinian corals Agaricia agaricites and Porites astreoides. Bull. Mar. Sci., 30, 883-887.

Borneman, E. (2001). Aquarium corals, selection, husbandry and natural history. Charlotte, USA: T.F.H. Publication.

Brown, B. E. (1997). Disturbances to Reefs in Recent Times. En: C. Birkeland (ed.), Life and Death of Coral Reefs (pp. 354-378). New York, USA: Chapman and Hall.

Caballero, H. y Guardia, E. (2003). Arrecifes de coral utilizados como zonas de colectas para exhibiciones en el Acuario Nacional de Cuba. I. Costa noroccidental de La Habana. Rev. Invest. Mar., 24(3), 205-220.

Caballero, H., Varona, G. y García, Y. (2004). Estructura ecológica de las comunidades de corales de Bahía de Cochinos, Cuba. Rev. Invest. Mar., 25(1), 23-26. 
Caballero, H., Rosales, D. y Alcalá, A. (2005). Estudio diagnóstico del arrecife coralino del Rincón de Guanabo, Ciudad de La Habana, Cuba. 1. Corales, esponjas y gorgonáceos. Rev. Invest. Mar., 26(3), 207-217.

Caballero, H., González-Ferrer, S., Cobián, D., Álvarez, S. y AlcoladoPrieto, P. (2007). Evaluación AGRRA del bentos de 10 sitios de buceo de María La Gorda, Bahía de Corrientes, Cuba. Rev. Invest. Mar., 28(2), 131-138.

Delgado, B. and Lapointe, E. (1994). Nutrient-limited productivity of calcareous versus fleshy macroalgae in an eutrophic, carbonate-rich tropical marine environment. Coral Reefs, 13, 151-159.

Elliot, J. M. (1971). Some methods of the statistical analysis of samples of benthic invertebrates. Freshwater Biological Association. Sci. Publ., 25, 124125.

Gardner, T. A., Côté, M. I., Gill, J. A., Grant, A. and Watkinson, A. R. (2003). Long-Term RegionWide Declines in Caribbean Corals. Science, 301, 958-960.

González-Ferrer, S. (2000). Caracterización de tres comunidades de coral del litoral norte de La Habana. Trabajo de Diploma. Universidad de La Habana.

González-Ferrer, S., Marcos, Z., Cantelar, K., Hernández-Zanuy, A., Martínez-Daranas, B., Valle, R., Abreu, M., Chávez, M. E., Muñoz, D., Ibar- zábal, D., Capetillo, N., Martínez, J. C. y Espinosa, J. (2004). Corales y organismos asociados en aguas cubanas. En: González-Ferrer (ed.), Corales pétreos, jardines sumergidos de Cuba (pp. 127-229). España: Editorial Academia.

González-Ferrer, S., Cantelar, K., Pina, F., Alcolado, P., Jiménez, A., Espinosa, J., Hernández, M. y Hernández, J. L. (2007a). Estado de los ecosistemas marinos y costeros, y algunas características ambientales y tendencias. En: P. M. Alcolado, E. E. García y M. Arellano-Acosta (eds.), Ecosistema Sabana Camagüey. Estado actual, avances y desafios en la protección y uso sostenible de la biodiversidad (pp. 38-45). Proyecto PNUD/GEF Sabana-Camagüey Cuba: Editorial Academia.

González-Ferrer, S., Caballero, H., Alcolado, P., Jiménez, A., Martín, F. y Cobián, D. (2007b). Diversidad de corales pétreos en 10 sitios de buceo recreativo de María La Gorda, Cuba. Rev. Invest. Mar., 28(2), 121-130.

González, P. (1999). Comunidades de esponjas, corales y gorgonias en un arrecife coralino costero de Ciudad de La Habana. Trabajo de Diploma. Universidad de La Habana.

Green, E. and Bruckner, W. A. (2000). The significance of coral disease epizootiology for coral reef conservation. Biol. Conserv., 96, 347-361. 
Guardia, E. y González-Sansón, G. (1997). Asociaciones de esponjas, gorgonias y corales de un arrecife en la costa noroccidental de Cuba III: Variación espacial de la diversidad. Rev. Invest. Mar., 18(3), 208-215.

Guardia, E., Angulo J., GonzálesSansón, G.., Aguilar C., GonzálezDías, P. (2004). Biodiversidad en la zona de buceo del Parque Nacional Punta Francés, Isla de la Juventud, Cuba. Rev. Invest. Mar., 25(2), 90-102.

Guardia, E., González-Díaz, P., Valdivia, A. y González-Sansón, G. (2005). Características generales de los arrecifes coralinos en la zona de buceo de Cayo Levisa, Archipiélago de los Colorados, Cuba. Rev. Invest. Mar., 26(1), 37-44.

Guzmán, H. B. y Guevara, C. A. (1998). Arrecifes coralinos de Bocas del Toro, Panamá: Distribución, estructura y estado de conservación de los arrecifes continentales de la laguna de Chiriquí y la Bahía Almirante. Revi. Biol. Trop., 46, 601-623.

Herrera, A. (1990). Efectos de la contaminación sobre las características de las comunidades bentónicas: El arrecife coralino. Tesis para la obtención del Grado de Candidato a Doctor en Ciencias Biológicas. Instituto de Oceanología de Cuba. Jones, L., Warner, G., Linton, D., Alcolado, P., Claro-Madruga, R., Clerveaux, W., Estrada, R.,
Fisher, T., Lockhart, K., Pardee, M., Pitt, J., Schelten, C. and Wild, R. (2004). Status of Coral Reefs in the Northern Caribbean and Atlantic Node of the GCRMN. En: C. Wilkinson (ed.), Status of the Coral Reefs of the World: 2004. Vol. 2 (pp. 451-472). Queensland, USA: GCRMN/ AIMS.

Jones, L., Alcolado, P. M., Cala, Y., Cobián, D., Coelho, V., Hernández, A., Jones, R., Mallela, J. and Manfrino, C. (2008). The Effects of Coral Bleac hing in the Northern Caribbean and Western Atlantic. En: C. Wilkinson y D. Souter (eds.), Status of Caribbean coral reefs after bleaching and hurricanes in 2005 (pp. 73-83). Townsville: Global Coral Reef Monitoring Network, and Reef and Rainforest Research Centre.

Kramer, P. (2003). Synthesis of coral reef health indicators for the western Atlantic: Result of the AGRRA program (1997-2000). Atoll Res. Bull., 46, 1-58.

Kramer, P. R. and Lang, J. C. (2003). The Atlanticand Gulf Rapid Reef Assessment (AGRRA) Protocols: former version 2.2. Appendix one. En: J. Lange (ed.), Status of Coral Reefs in the Western Atlantic: Results of Inicial Surveys, Atlantic and Gula Rapad Reef Assessment (AGRRA) Program. Atoll. Res. Bull., 496, 611-624.

Lang, J., Alcolado, P. M., CarricartGanivet, P., Chiappone, M., 
Curran, A., Dustan, P., Gaudian, G., Geraldes, F., Gittings, S., Smith, R., Tunnell, W. and Wiener, J. (1998). Status of coral reefs in the northern areas of the Wider Caribbean. En: C. Wilkinson (ed.), Status of coral reefs of the world: 1998 (pp. 123-134). Australian Institute of Marine Science. Australia: Global Coral Reef Monitoring Network.

Lapointe, B. E. (1997). Nutrient thresholds for bottom-up control of macroalgal blooms on coral reefs in Jamaica and southeast, Florida. Limnol. Oceanograph., 42, 1119-1131.

Lapointe, B. E., Littler, M. M. and Littler, D. S. (1997). Macroalgal overgrowth of fringing coral reefs at Discovery Bay, Jamaica: bottom-up control versus topdown control. Proc. 8th Int. Sym. Coral Reefs, 1, 927-932.

Lluis-Riera, M. (1983). Estudios hidrológicos de la plataforma noroccidental de Cuba (zona c). Rep. Invest. Inst. Oceanología, 13, 1-33.

Martínez-Estalella, N. y Herrera, A. (1989). Estudio de la comunidad coralina del arrecife del Rincón de Guanabo, Cuba. Academia de Ciencias de Cuba. Instituto de Oceanología. Rep. Invest. Inst. Oceanología, 9, 28 p.

McClanahan, T. R. (2004). Phosphorous andnitrogenus enrichement do not enhance brown frondose "macroalgae". Mar. Poll, Bull., 48, 196-199.

Muller-Parker, G. and D'Elia, C. F. (1997). Interaction between corals and their symbiotic algae. En: C. Birkeland (ed.), Life and death of coral reefs (pp. 96-112). New York, USA: Chapman and Hall.

Pielou, E. C. (1966). Shannon's formula as a measurement of specific diversity: its use and mesure. Am. Nat., 100, 463-465.

Richmond, R. H. (1997). Reproduction and recruitment in corals. En: C. Birkeland (ed.), Life and death of coral reefs (pp. 175196). New York, USA: Chapman and Hall.

Schuttenberg, H. and Marshall, P. (2008). Managing for Mass Coral Bleaching: Strategies for Suppor-ting Socio-ecological Resilience. Townsville: Global Coral Reef Monitoring Network, and Reef and Rainforest Research Centre.

Shannon, C. E. \& Weaver, W. (1963). The mathematical theory of communication. En: J. A. Ludwig (ed.), Statistical Ecology. A primer on methods and computing. A WileyInterscience publication. $337 \mathrm{p}$.

Steneck, R. S., Lang, J. C, Kramer, P. A. and Ginsburg, R. N. (1997). AGRA, Rapid Assessment Protocol. http// coral. Amol. Noaa. Gov/agra/agr1.html. 
Taylor, W. R. (1961). Aggregation variance and the mean. Nature, 189(4766), 732-735.

Torres, J. and Morelock, J. (2002). Effect of Terrigenous Sediment Influx on Coral Cover and Rates of Three Caribbean Massive Coral Species. J. Caribbean Sci., 1(38), 222-229.

Valdivia, A. y Guardia, E. (2004). Estructura de la comunidad de corales en el arrecife costero de Boca de Canasí, La Habana, Cuba. Rev. Invest. Mar., 25(1), 15-22.

Wilkinson, C. and Souter, D. (2008). Status of Caribbean coral reefs after bleaching and hurricanes in 2005.
Townsville: Global Coral Reef Monitoring Network, and Reef and Rainforest Research Centre.

Woodley, J., Alcolado, P., Austin, T., Barnes, J., Claro-Madruga, R., Ebanks-Petrie, G., Estrada, R., Geraldes, F., Glasspool, A., Homer, F., Luckhurst, B., Phillips, E., Shim, D., Smith, R., Sullivan-Sealey, K., Vega, M., Ward, J. and Wiener, J. (2000). Status of coral reefs in the northern Caribbean and western Atlantic. En: C. Wilkinson (ed.), Status of coral reefs of the world (pp. 239-260). Australian Institute of Marine Science: Global Coral Reef Monitoring Network. 